TRANSACTIONS OF THE

AMERICAN MATHEMATICAL SOCIETY

Volume 351, Number 9, Pages 3743-3755

S 0002-9947(99)02501-5

Article electronically published on April 27, 1999

\title{
ON THE $L^{2} \rightarrow L^{\infty}$ NORMS OF SPECTRAL MULTIPLIERS OF "QUASI-HOMOGENEOUS" OPERATORS ON HOMOGENEOUS GROUPS
}

\author{
ADAM SIKORA
}

\begin{abstract}
We study the $L^{2} \rightarrow L^{\infty}$ norms of spectral projectors and spectral multipliers of left-invariant elliptic and subelliptic second-order differential operators on homogeneous Lie groups. We obtain a precise description of the $L^{2} \rightarrow L^{\infty}$ norms of spectral multipliers for some class of operators which we call quasi-homogeneous. As an application we prove a stronger version of Alexopoulos' spectral multiplier theorem for this class of operators.
\end{abstract}

\section{INTRODUCTION}

Let $G$ be a nilpotent Lie group with Lie algebra $\mathfrak{g}$. For a system $X_{1}, \ldots, X_{k} \in \mathfrak{g}$ of left-invariant vector fields on $G$ satisfying Hörmander's condition we define an operator $L$ by the formula

$$
L=-\sum_{i=1}^{k} X_{i}^{2} .
$$

It is well known that the closure of the operator $L$ in $L^{2}(G)$ is self-adjoint. Thus it admits a spectral resolution

$$
L=\int_{0}^{\infty} \lambda d E_{L}(\lambda)
$$

For any bounded Borel function $F$ we define an operator $F(L)$ by the formula

$$
F(L)=\int_{0}^{\infty} F(\lambda) d E_{L}(\lambda)
$$

By $K_{F(L)}$ we denote the kernel of the operator $F(L)$, i.e. a distribution such that

$$
F(L) \psi=\psi * K_{F(L)} .
$$

In the present paper we investigate the $L^{2}(G) \rightarrow L^{\infty}(G)$ norms of spectral multipliers of the operator $L$ :

$$
\|F(L)\|_{L^{1} \rightarrow L^{2}}^{2}=\|F(L)\|_{L^{2} \rightarrow L^{\infty}}^{2}=\left\|K_{F(L)}\right\|_{L^{2}}^{2} .
$$

In the case of elliptic differential and pseudo-differential self-adjoint operators on a compact manifold $M$ the $L^{2} \rightarrow L^{\infty}$ norms of spectral multipliers were investigated by Hörmander [10], [9], by Sogge [16], [17], [18] and by Christ and Sogge in [3]. In [10] Hörmander proved that if $\chi_{[a, b)}$ denotes the characteristic function of an

Received by the editors November 10, 1996.

1991 Mathematics Subject Classification. Primary 42B15; Secondary 43A22, 35P99.

(C)1999 American Mathematical Society 
interval $[a, b)$ and $P$ is a self-adjoint elliptic differential operator of order $m$, then (see Lemma 4.3 on p. 211 of [10])

$$
\left\|\chi_{\left[(k-1)^{m}, k^{m}\right)}(P)\right\|_{L^{2} \rightarrow L^{\infty}}^{2} \leq C k^{d-1},
$$

where $d$ is the dimension of the manifold $M$. In [18] Sogge proved even more precise estimates for spectral projectors of an elliptic differential operator of second order $P$ (see Lemma 2.2 of $[16]$ ):

$$
\left\|\chi_{\left[(k-1)^{2}, k^{2}\right)}(P)\right\|_{L^{p} \rightarrow L^{2}} \leq C k^{\alpha(p)},
$$

for each $1 \leq p<2$ such that $1 / p-1 / 2 \geq 1 /(d+1)$, where

$$
\alpha(p)=\max \{0, d|1 / p-1 / 2|-1 / 2\} .
$$

In [3] Christ and Sogge proved that if $P$ is a first order elliptic self-adjoint pseudodifferential operator on a compact manifold with positive eigenvalues then

$$
\left\|\chi_{[k-1, k)}(P)\right\|_{L^{2} \rightarrow L^{\infty}}^{2} \leq C k^{d-1} .
$$

The estimates (3),(4) and (5) are used in the above papers to obtain several results concerning the convergence in $L^{p}(M)$ of Riesz means for $P$ and spectral multiplier theorems for the operator $P$.

In the case of operators on unimodular Lie groups a useful way of describing the $L^{2} \rightarrow L^{\infty}$ norm of spectral multipliers and projectors is the following analogue of Plancherel's measure (compare Proposition 3 of [2]).

Lemma 1. If we define the measure $d \mu_{L}$ by the formula

$$
\int_{0}^{\infty} F(\lambda) d \mu_{L}(\lambda)=\int_{0}^{\infty} F(\lambda) e^{-2 \lambda} d\left(E(\lambda) p_{1}, p_{1}\right),
$$

then

$$
\|F(L)\|_{L^{1} \rightarrow L^{2}}^{2}=\|F(L)\|_{L^{2} \rightarrow L^{\infty}}^{2}=\left\|K_{F(L)}\right\|_{L^{2}}^{2}=\int_{\mathbf{R}}|F(\lambda)|^{2} d \mu_{L}(\lambda),
$$

where $p_{t}$ is the heat kernel corresponding to the self-adjoint elliptic left-invariant operator L, i.e. $p_{t}=K_{\exp (-t L)}$.

Proof. Indeed,

$$
\begin{gathered}
\left\|K_{F(L)}\right\|_{L^{2}}=\int d\left(E(\lambda) K_{F(L)}, K_{F(L)}\right) \\
=\int e^{2 \lambda} d\left(E(\lambda) \exp (-L)\left(K_{F(L)}\right), \exp (-L)\left(K_{F(L)}\right)\right) \\
=\int e^{2 \lambda} d\left(E(\lambda) F(L)\left(p_{1}\right), F(L)\left(p_{1}\right)\right) \\
=\int|F(\lambda)|^{2} e^{2 \lambda} d\left(E(\lambda) p_{1}, p_{1}\right) .
\end{gathered}
$$

Following Christ [2] and Alexopoulos [1] we will call $d \mu_{L}$ the Plancherel measure of the operator $L$.

Now let $G$ be a nilpotent homogeneous Lie group with Lie algebra $\mathfrak{g}$ and let $\delta_{t}: G \rightarrow G$ for $t>0$ be a family of dilations of the group $G$. (We refer to [6] for the definition of a homogeneous group and dilations.) For homogeneous groups the exponential mapping identifies $G$ with its Lie algebra $\mathfrak{g}$, so we may regard the $\delta_{t}$ 
instead as maps from $\mathfrak{g}$ into itself. They are Lie algebra automorphisms. Next, for an arbitrary function $\psi$ on $G$ and $t>0$ we set

$$
\bar{\delta}_{t} \psi(x)=\psi\left(\delta_{t} x\right) .
$$

We say that an operator $L$ of the form (1) is homogeneous if

$$
L \circ \bar{\delta}_{t}=t^{2} \bar{\delta}_{t} \circ L
$$

or equivalently, if there are vectors $X_{1}, \ldots, X_{k}$ such that

$$
L=-\sum_{i=1}^{k} X_{i}^{2}
$$

and

$$
\delta_{t} X_{i}=t X_{i} \text { for } i=1, \ldots, k .
$$

An operator $L$ will be called quasi-homogeneous if there exist vectors $X_{1}, \ldots, X_{k}$ such that $L$ is defined by (7) and

$$
\delta_{t} X_{i}=t^{d_{i}} X_{i} \text { for } i=1, \ldots, k,
$$

where $1 \leq d_{1} \leq \ldots \leq d_{k}$. It is not difficult to compute the Plancherel measure and therefore also obtain a precise description of the $L^{2} \rightarrow L^{\infty}$ norms of spectral projectors and multipliers for a homogeneous operator. In [2] Christ shows that if $L$ is a homogeneous operator defined by (1), then for some constant $C$ (Proposition 3 of $[2])$

$$
d \mu_{L}(\lambda)=C \lambda^{Q / 2-1} d \lambda,
$$

where $Q$ is the homogeneous dimension of the group $G$. Christ applied (9) in the proof of the multiplier theorem for the operator $L$ (Theorem 1 of [2]). In the present paper we prove that for 'quasi-homogeneous' operators the Plancherel measure has smooth density satisfying estimates similar to that in (9).

In [1] Alexopoulos obtained a multiplier theorem for all second order sub-elliptic operators on Lie groups of polynomial growth. However, Alexopoulos' theorem applied to a homogeneous operator gives a weaker result then Christ's multiplier theorem. Alexopoulos suggested that he lost some exponents in his work because of lack of an analogue of equation (9) for the Plancherel measure for the operators that he was studying. Indeed, as an application we will use our estimates for the Plancherel measure of quasi-homogeneous operators to obtain a stronger version of Alexopoulos' multiplier theorem for such operators.

\section{Smoothness of the density of Plancherel measure FOR QUASI-HOMOGENEOUS OPERATORS}

For every operator $L$ defined by (1) on a nilpotent Lie group there exist natural numbers $d$ and $D$ such that

$$
\left\|p_{t / 2}\right\|_{L^{2}}^{2}=p_{t}(e) \sim \begin{cases}t^{-d / 2} & \text { if } t \leq 1, \\ t^{-D / 2} & \text { if } t>1 .\end{cases}
$$

See, for example, [20]. Following [20], we will call $d$ and $D$ the dimension of the heat kernel at zero and at infinity, respectively. If the group $G$ is simply connected, then $d \leq D$; see [20], Chapter IV.5. Every homogeneous group is simply connected; 
thus $d \leq D$ for all operators which we investigate here. Note that if the heat kernel corresponding to an operator $L$ satisfies (10), then

$$
\begin{aligned}
\mu_{L}([0, r)) & \leq e \int_{0}^{r} d \mu_{L}(\lambda) e^{-\lambda r^{-1}} \leq e \int_{0}^{\infty} d \mu_{L}(\lambda) e^{-\lambda r^{-1}} \\
& =e\left\|p_{\frac{1}{2 r}}(e)\right\|_{L^{2}}^{2} \leq C\left(r^{d / 2} \wedge r^{D / 2}\right) .
\end{aligned}
$$

For quasi-homogeneous operators we can prove much sharper estimates. The following improvement of (11) is the main result of this paper.

Theorem 1. Suppose that the operator $L$ defined by (7) is quasi-homogeneous. Then the measure $\mu_{L}$ is absolutely continuous with respect to the Lebesgue measure $d \lambda$ :

$$
d \mu_{L}=\alpha(\lambda) d \lambda
$$

The function $\alpha: \mathbf{R}_{+} \rightarrow \mathbf{R}$ is smooth, and

$$
\left|\frac{d^{n} \alpha}{d \lambda^{n}}(\lambda)\right| \leq C_{n} \begin{cases}\lambda^{d / 2-(n+1)} & \text { if } \lambda \leq 1 \\ \lambda^{D / 2-(n+1)} & \text { if } \lambda>1\end{cases}
$$

We divide the proof of Theorem 1 into a few lemmas. First for complex numbers $a_{i}, i=1, \ldots, k$, such that $\operatorname{Re} a_{i}>0$, we define operator $L^{a_{1}, \ldots, a_{k}}$ by the formula

$$
L^{a_{1}, \ldots, a_{k}}=\sum_{i=1}^{k} a_{i} X_{i}^{2} .
$$

Of course $L^{1, \ldots, 1}=L$. By virtue of Proposition 3.7 and Theorem 4.1 of [5] the operator $L^{a_{1}, \ldots, a_{k}}$ generates a semigroup on $L^{p}$ for all $1 \leq p \leq \infty$. By $P_{t}^{a_{1}, \ldots, a_{k}}$ we will denote the semigroup generated by the operator $L^{a_{1}, \ldots, a_{k}}$ and by $p_{t}^{a_{1}, \ldots, a_{k}}$ we will denote the kernel of the operator $P_{t}^{a_{1}, \ldots, a_{k}}$. It is proved in [5] (Theorem 4.1) that $p_{t}^{a_{1}, \ldots, a_{k}} \in C^{\infty}(G)$ and that for some constants $a, b, \omega>0$

$$
\left|p_{t}^{a_{1}, \ldots, a_{k}}(g)\right| \leq a t^{-d / 2} e^{\omega t} \exp \left(-b \frac{|g|^{2}}{t}\right),
$$

where $|g|$ is the distance from the identity $e$ to the element $g$ of $G$ in the optimal control metric corresponding to the system $X_{1}, \ldots, X_{k}$. In the sequel we will need a different kind of estimate for $p_{t}^{a_{1}, \ldots, a_{k}}$.

Lemma 2. Let $p_{t}^{a_{1}, \ldots, a_{k}}$ be the kernel corresponding to the operator $L^{a_{1}, \ldots, a_{k}}$. Then

$$
\left|\frac{d^{n}}{d t^{n}} p_{t}^{a_{1}, \ldots, a_{k}}(e)\right| \leq\left\|\frac{d^{n}}{d t^{n}} p_{t}^{a_{1}, \ldots, a_{k}}\right\|_{L^{\infty}} \leq C_{n}^{\prime} \begin{cases}t^{-d / 2-n} & \text { if } t \leq 1 \\ t^{-D / 2-n} & \text { if } t>1 .\end{cases}
$$

Proof. First we show that the semigroup $P_{t}^{a_{1}, \ldots, a_{k}}$ is equicontinuous on $L^{1}(G)$ and $L^{\infty}(G)$. Indeed, let $\mathfrak{g}^{\prime}$ be a free nilpotent Lie algebra with $k$ generators $Z_{1}, \ldots, Z_{k}$ and the same rank as the algebra $\mathfrak{g}$. There exists a homeomorphism $d U: \mathfrak{g}^{\prime} \rightarrow \mathfrak{g}$ such that

$$
d U\left(Z_{i}\right)=X_{i} \text { for } i=1, \ldots, k
$$

(See [20], p. 46.) Next let $U: G^{\prime} \rightarrow G$ be the corresponding homeomorphism of the groups $G^{\prime}$ and $G$. By $p_{t}^{\prime}$ we denote the heat kernel corresponding to the operator

$$
L^{\prime}=-\sum_{i=1}^{k} a_{i} Z_{i}^{2} .
$$


$\mathfrak{g}^{\prime}$ is a free nilpotent Lie algebra, so there exists a family of dilations $\delta_{t}^{\prime}$ on $\mathfrak{g}^{\prime}$ such that

$$
\delta_{t}^{\prime} Z_{i}=t Z_{i}
$$

Note that $L^{\prime}$ is a homogeneous operator with respect to $\delta_{t}^{\prime}$, so

$$
\exp \left(-t L^{\prime}\right)=\bar{\delta}_{t^{-1 / 2}}^{\prime} \circ \exp \left(-L^{\prime}\right) \circ \bar{\delta}_{t^{1 / 2}}^{\prime}
$$

and

$$
p_{t}^{\prime}(x)=t^{-Q^{\prime} / 2} p_{1}^{\prime}\left(\delta_{t^{-1 / 2}}^{\prime} x\right),
$$

where $Q^{\prime}$ is the homogeneous dimension of the group $G^{\prime}$. By (14)

$$
\left\|p_{t}^{\prime}\right\|_{L^{1}}=\left\|p_{1}^{\prime}\right\|_{L^{1}}<\infty
$$

However, we note that for any Borel set $A \subset G$

$$
\int_{A} p_{t}^{a_{1}, \ldots, a_{k}} d x=\int_{U^{-1}(A)} p_{t}^{\prime} d x^{\prime}
$$

Thus $\left\|p_{t}^{a_{1}, \ldots, a_{k}}\right\|_{L^{1}(G)} \leq\left\|p_{t}^{\prime}\right\|_{L^{1}\left(G^{\prime}\right)}$, and for all $1 \leq p \leq \infty$

$$
\left\|P_{t}^{a_{1}, \ldots, a_{k}}\right\|_{L^{p} \rightarrow L^{p}} \leq\left\|p_{t}^{a_{1}, \ldots, a_{k}}\right\|_{L^{1}} \leq\left\|p_{1}^{\prime}\right\|_{L^{1}}<\infty .
$$

By (15) the semigroup $P_{t}^{a_{1}, \ldots, a_{k}}$ is equicontinuous on all $L^{p}(G)$. Hence by virtue of Theorem II.3.2 of [20], to show (13) for $n=0$ it is enough to prove that $L^{a_{1}, \ldots, a_{k}}$ satisfies the following Nash inequalities:

$$
\begin{gathered}
\|\psi\|_{L^{2}}^{2+2 / d} \leq C \operatorname{Re}\left(L^{a_{1}, \ldots, a_{k}} \psi, \psi\right)\|\psi\|_{L^{1}}^{4 / d}, \\
\|\psi\|_{L^{2}}^{2+2 / D} \leq C \operatorname{Re}\left(L^{a_{1}, \ldots, a_{k}} \psi, \psi\right)\|\psi\|_{L^{1}}^{4 / D} .
\end{gathered}
$$

If we put $\alpha_{i}=\operatorname{Re} a_{i}, Y_{i}=\alpha_{i}^{1 / 2} X_{i}$ and

$$
L^{\prime \prime}=-\sum_{i=1}^{k} Y_{i}^{2}
$$

then

$$
\operatorname{Re}\left(L^{a_{1}, \ldots, a_{k}} \psi, \psi\right)=\left(L^{\prime \prime} \psi, \psi\right) .
$$

However, $L^{\prime \prime}$ generates a symmetric Markov semigroup, and it is well known that (16) and (17) hold for $L^{\prime \prime}$. See e.g. Chapter IV of [20] (see also Theorem 2.4.6 of [4]). To get (13) for all $n$ we note that $P^{a_{1}, \ldots, a_{k}}$ is a bounded analytic semigroup on $L^{1}$, so

$$
\left\|\left(L^{a_{1}, \ldots, a_{k}}\right)^{n} P_{t}^{a_{1}, \ldots, a_{k}}\right\|_{L^{1} \rightarrow L^{1}} \leq C_{n}^{\prime \prime} t^{-n} .
$$

However,

$$
\frac{d^{n}}{d t^{n}} P_{t}^{a_{1}, \ldots, a_{k}}=\left(L^{a_{1}, \ldots, a_{k}}\right)^{n} P_{t}^{a_{1}, \ldots, a_{k}}
$$

and

$$
\begin{gathered}
\left\|\frac{d^{n}}{d t^{n}} p_{t}^{a_{1}, \ldots, a_{k}}\right\|_{L^{\infty}}=\left\|\left(L^{a_{1}, \ldots, a_{k}}\right)^{n} P_{t}^{a_{1}, \ldots, a_{k}}\right\|_{L^{1} \rightarrow L^{\infty}} \\
\leq\left\|\left(L^{a_{1}, \ldots, a_{k}}\right)^{n} P_{t / 2}^{a_{1}, \ldots, a_{k}}\right\|_{L^{1} \rightarrow L^{1}} \times\left\|P_{t / 2}^{a_{1}, \ldots, a_{k}}\right\|_{L^{1} \rightarrow L^{\infty}} \\
\leq C_{n}^{\prime} t^{-n}\left\|P_{t / 2}^{a_{1}, \ldots, a_{k}}\right\|_{L^{1} \rightarrow L^{\infty}} .
\end{gathered}
$$


Hence (13) for arbitrary $n \geq 0$ follows from the case $n=0$. This proves Lemma 2 .

Next, note that if $L$ is quasi-homogeneous, then by (8) for $t>0$ we have

$$
\bar{\delta}_{1 / t} \circ L^{a_{1}, \ldots, a_{k}} \circ \bar{\delta}_{t}=L^{t^{2 d_{1}} a_{1}, \ldots, t^{2 d_{k}} a_{k}} .
$$

Thus

$$
\bar{\delta}_{1 / t} \circ P_{s}^{a_{1}, \ldots, a_{k}} \circ \bar{\delta}_{t}=P_{s}^{t^{2 d_{1}} a_{1}, \ldots, t^{2 d_{k}} a_{k}} .
$$

By virtue of (18),

$$
\frac{1}{t^{Q}} p_{s}^{a_{1}, \ldots, a_{k}}\left(\delta_{t^{-1}} g\right)=p_{s}^{t^{2 d_{1}} a_{1}, \ldots, t^{2 d_{k}} a_{k}}(g)
$$

but if $e$ is the identity element of $G$ then $\delta_{t^{-1}} e=e$, so

$$
\frac{1}{t^{Q}} p_{s}^{a_{1}, \ldots, a_{k}}(e)=p_{s}^{t^{2 d_{1}} a_{1}, \ldots, t^{2 d_{k}} a_{k}}(e) \text {. }
$$

The following lemma shows that (19) is valid for $t \in \Sigma$, where $\Sigma$ is a certain sector in the complex plane containing the nonnegative real numbers.

Lemma 3. For complex numbers $a_{i}, i=1, \ldots, k$, such that $\operatorname{Re} a_{i}>0$, the semigroups $P_{t}^{a_{1}, \ldots, a_{k}}$ depend analytically on the coefficients $a_{1}, \ldots, a_{k}$. Hence

$$
\frac{1}{t^{Q}} p_{s}^{a_{1}, \ldots, a_{k}}(e)=p_{s}^{t^{2 d_{1}} a_{1}, \ldots, t^{2 d_{k}} a_{k}}(e) .
$$

for all $t \in \Sigma_{a, b}$, where $\Sigma_{a, b}=\{z \in \mathbf{C}: \quad b<\arg z<a\}, a=\min \frac{\pi / 2-\arg a_{i}}{2 d_{i}}$ and $b=\max \frac{-\pi / 2-\arg a_{i}}{2 d_{i}}$.

Proof. We note that the resolvent of the operator $L^{a_{1}, \ldots, a_{k}}$,

$$
R\left(\lambda, L^{a_{1}, \ldots, a_{k}}\right)=\left(\lambda I-L^{a_{1}, \ldots, a_{k}}\right)^{-1}
$$

is an analytic function of $a_{1}, \ldots, a_{k}$. Indeed,

$$
R\left(\lambda, L^{a_{1}, \ldots, a_{k}}+z X_{i}^{2}\right)=R\left(\lambda, L^{a_{1}, \ldots, a_{k}}\right)\left(\sum_{k=0}^{\infty}\left(z X_{i}^{2} R\left(\lambda, L^{a_{1}, \ldots, a_{k}}\right)\right)^{k}\right),
$$

and

$$
\partial_{a_{i}} R\left(\lambda, L^{a_{1}, \ldots, a_{k}}\right)=R\left(\lambda, L^{a_{1}, \ldots, a_{k}}\right) X_{i}^{2} R\left(\lambda, L^{a_{1}, \ldots, a_{k}}\right) .
$$

However by virtue of Theorem 3.3 in Chapter II of [5], $X_{i}^{2} R\left(\lambda, L^{a_{1}, \ldots, a_{k}}\right)$ is a bounded operator on $L^{2}$ and $R\left(\lambda, L^{a_{1}, \ldots, a_{k}}\right)$ is an analytic function of $a_{1}, \ldots, a_{k}$. Now by the following formula ([13], Chapter 3, Lemma 4.1)

$$
\begin{gathered}
R\left(\lambda, L^{a_{1}, \ldots, a_{k}}\right)\left[P_{t}^{a_{1}, \ldots, a_{k}}-P_{t}^{b_{1}, \ldots, b_{k}}\right] R\left(\lambda, L^{b_{1}, \ldots, b_{k}}\right) \\
=\int_{0}^{t} P_{(t-s)}^{a_{1}, \ldots, a_{k}}\left[R\left(\lambda, L^{a_{1}, \ldots, a_{k}}\right)-R\left(\lambda, L^{b_{1}, \ldots, b_{k}}\right)\right] P_{s}^{b_{1}, \ldots, b_{k}} d s
\end{gathered}
$$

we have

$$
\begin{gathered}
R\left(\lambda, L^{a_{1}, \ldots, a_{k}}\right) \partial_{a_{i}} P_{t}^{a_{1}, \ldots, a_{k}} R\left(\lambda, L^{a_{1}, \ldots, a_{k}}\right) \\
=\int_{0}^{t} P_{(t-s)}^{a_{1}, \ldots, a_{k}} \partial_{a_{i}} R\left(\lambda, L^{a_{1}, \ldots, a_{k}}\right) P_{s}^{a_{1}, \ldots, a_{k}} d s \\
=\int_{0}^{t} P_{(t-s)}^{a_{1}, \ldots, a_{k}} R\left(\lambda, L^{a_{1}, \ldots, a_{k}}\right) X_{i}^{2} R\left(\lambda, L^{a_{1}, \ldots, a_{k}}\right) P_{s}^{a_{1}, \ldots, a_{k}} d s .
\end{gathered}
$$


This proves that the semigroup $P^{a_{1}, \ldots, a_{k}}$ is an analytic function of $a_{1}, \ldots, a_{k}$, so both sides of (20) are analytic functions of $t$. Hence to prove (20) it is enough to recall that, by virtue of (19), (20) is true if $t$ is real and $t>0$.

Finally, to prove Theorem 1 we will need the following lemma.

Lemma 4. Suppose $p_{z}$ is a heat kernel corresponding to the quasi-homogeneous operator. Then the value of the kernel at the origin $p_{z}(e)$, regarded as a function of the variable $z$, extends analytically to the sector $\Sigma_{\epsilon+\pi / 2}$ for some $\epsilon>0$. Moreover, for $z=i s$, where $s \in \mathbf{R}$,

$$
\left|\frac{d^{n}}{d z^{n}} p_{z}(e)\right| \leq c_{n} \begin{cases}|z|^{-d / 2-n} & \text { if }|z| \leq 1 \\ |z|^{-D / 2-n} & \text { if }|z|>1\end{cases}
$$

Proof. We put $t=e^{-i \theta}, \theta=\frac{\pi}{4 d_{k}}$, and $z=r e^{i \omega}, r>0$. Now $p_{z}(e)=p_{z}^{1, \ldots, 1}(e)$ is an analytic function of $z$ for $-\pi / 2<\omega<\pi / 2$. On the other hand, $p_{z}^{t^{2 d_{1}}, \ldots, t^{2 d_{k}}}(e)$ $=p_{r}^{e^{i \omega} t^{2 d_{1}}, \ldots, e^{i \omega} t^{2 d_{k}}}(e)$ is an analytic function of $z$ for

$$
0<\omega<\pi / 2+\theta \times 2 \min \left\{d_{1}, \ldots, d_{k}\right\}=\pi / 2+\pi \frac{d_{1}}{2 d_{k}} .
$$

However, by virtue of Lemma 3 , for $0<\omega<\pi / 2$ we have

$$
\frac{1}{t^{Q}} p_{z}^{1, \ldots, 1}(e)=\frac{1}{t^{Q}} p_{r}^{e^{i \omega}, \ldots, e^{i \omega}}(e)=p_{r}^{e^{i \omega} t^{2 d_{1}}, \ldots, e^{i \omega} t^{2 d_{k}}}(e)=p_{z}^{t^{2 d_{1}}, \ldots, t^{2 d_{k}}}(e) .
$$

This gives us an analytic extension of $p_{z}(e)$ for $-\pi / 2<\omega<\pi / 2+\pi \frac{d_{1}}{4 d_{k}}$. Finally, since Re $i t^{2 d_{j}}>0$, by Lemma 2 for $\omega=\pi / 2$ we have

$$
\begin{aligned}
\left|\frac{d^{n}}{d z^{n}} p_{z}(e)\right| & \leq\left\|t^{Q} \frac{d^{n}}{d z^{n}} p_{z}^{t^{2 d_{1}}, \ldots, t^{2 d_{k}}}\right\|_{L^{\infty}}=\left\|t^{Q} \frac{d^{n}}{d r^{n}} p_{r}^{i t^{d_{1}}, \ldots, i t^{2 d_{k}}}\right\|_{L^{\infty}} \\
& \leq c_{n}\left(r^{-d / 2-n} \wedge r^{-D / 2-n}\right)=c_{n}\left(|z|^{-d / 2-n} \wedge|z|^{-D / 2-n}\right),
\end{aligned}
$$

which proves (21) for $\omega=\pi / 2$. In the same way we can construct an analytic extension of $p_{z}(e)$ for $-\pi / 2-\pi \frac{d_{1}}{4 d_{k}}<\omega<\pi / 2$ and prove (21) for $\omega=-\pi / 2$. This finishes the proof of Lemma 4.

Proof of Theorem 1. Note that if $K_{F(L)}$ is defined by (2) and $\mu_{L}$ is the Plancherel measure for $L$, then

$$
K_{F(L)}(e)=\int_{0}^{\infty} F(\lambda) d \mu_{L}(\lambda)
$$

and so

$$
p_{z}(e)=\int_{0}^{\infty} e^{z \lambda} d \mu_{L}(\lambda) .
$$

Hence $p_{-i t}(e)=\hat{\mu}(t)$. Now Theorem 1 follows from

Proposition 1. Assume that for any natural number $n$ the Fourier transform of function $f: \mathbf{R} \rightarrow \mathbf{C}$ satisfies the estimate

$$
\left|\frac{d^{n}}{d t^{n}} \hat{f}(t)\right| \leq c_{n}^{\prime} t^{a-n}
$$

and that $\operatorname{supp} f \subset[0, \infty)$. Then

$$
\left|\frac{d^{n}}{d t^{n}} f(t)\right| \leq c_{n}^{\prime \prime} t^{-1-a-n}
$$

for any $n \leq 0$. 
Proof. We define the function $x_{+}^{1 / 2}$ by the formula

$$
x_{+}^{-1 / 2}(x)= \begin{cases}x^{-1 / 2} & \text { if } x \leq 0 \\ 0 & \text { if } x<0\end{cases}
$$

If $f$ is a distribution such that supp $f \subset[0, \infty)$, then $f * x_{+}^{-1 / 2}$ is well defined (see (4.2.6) on p. 104 of [8]) and

$$
\frac{1}{(\Gamma(1 / 2))^{2}} \frac{d}{d t} f * x_{+}^{-1 / 2} * x_{+}^{-1 / 2}=f
$$

Next we note that (see example 7.1.17 of [8])

$$
\left|\left(f * x_{+}^{-1 / 2}\right)^{\wedge}(\lambda)\right|=\Gamma(1 / 2)\left|\hat{f}(\lambda) \lambda^{-1 / 2}\right|
$$

and that if supp $f \subset[0, \infty)$ and $|f(x)| \leq c|t|^{b}$ for $b>-1$, then

$$
\left|f * x_{+}^{-1 / 2}(t)\right| \leq c^{\prime}|t|^{b+1 / 2}
$$

Hence it is enough to prove Proposition 1 for $a>-1$. But this can be proved in the same way as Proposition 2 on p. 245 of [19]. This finishes the proof of Theorem 1.

Remark 1. If $\Delta$ is a standard Laplace operator on the one-dimensional torus, then it is easy to check that

$$
\mu_{\Delta}=\delta_{0}+2 \sum_{j=1}^{\infty} \delta_{j^{2}} .
$$

Of course $\mu_{\Delta}$ does not have a density, so Theorem 1 is not true for all nilpotent Lie groups. However we are not aware of any counterexample to Theorem 1 in case of a simple connected group. Also, we do not know if Hörmander's estimates (3) are true for all nilpotent Lie groups.

Remark 2. It is interesting to note that by carefully reading the proof of Theorem 1 we can easily show that the function $\alpha(z)$ can be extend analytically to a sector $\Sigma_{\varepsilon}$ for some $\varepsilon>0$ and its extension satisfies (12).

Remark 3. Suppose that $X_{1}, \ldots, X_{k}$ is homogeneous algebraic base of $\mathfrak{g}$, i.e., $\mathfrak{g}$ is generated by $X_{1}, \ldots, X_{k}$ as a Lie algebra and (8) is satisfied. Next let an operator $L$ be defined by the formula

$$
L=\sum_{i=1}^{k} \sum_{i=1}^{k} a_{i j} X_{i} X_{j},
$$

where $\left(a_{i j}\right)$ is a positive definite, symmetric matrix. It follows from the proof that Theorem 1 holds for $L$ if the matrix $\left(a_{i j}\left(d_{i}+d_{j}\right)\right)$ is positive definite.

\section{Multiplier THEOREM}

In this section we apply the estimates for the Plancherel measure of quasihomogeneous operators from Theorem 1 to obtain a stronger version of Alexopoulos' multiplier theorem [1]. In what follows, for $g \in G$ we put $|g|=\rho(e, g)$, where $e$ is the identity element of $G$ and $\rho$ is the optimal control metric corresponding to the system $X_{1}, \ldots, X_{k}$. By $|x|$ we denote the Euclidean norm of $x \in \mathbf{R}^{Q}$. We assume that $L$ is a quasi-homogeneous operator defined by (1). We start this section with the following lemma. 
Lemma 5. Suppose that the Plancherel measure of L satisfies $d \mu_{L}=\alpha_{L}(\lambda) d \lambda$ and

$$
\alpha_{L}(\lambda) \leq C \lambda^{Q / 2-1} .
$$

Then for any bounded Borel function $F: \mathbf{R} \rightarrow \mathbf{C}$ we have

$$
\int_{G}|g|^{a}\left|K_{F(\sqrt{L})}(g)\right|^{2} d g \leq C^{\prime} \int_{\mathbf{R}^{Q}}|x|^{a}\left|K_{F\left(\sqrt{\Delta_{Q}}\right)}(x)\right|^{2} d x
$$

and

$$
\int_{G}|g|^{a}\left|X_{i} * K_{F(\sqrt{L})}\right|^{2} d g \leq C^{\prime} \int_{\mathbf{R}^{Q+2}}|x|^{a}\left|K_{F\left(\sqrt{\Delta_{Q+2}}\right)}\right|^{2} d x,
$$

where $\Delta_{Q}$ is a standard Laplace operator on $\mathbf{R}^{Q}$.

Lemma 5 for $d \mu_{L}=C \lambda^{Q / 2-1} d \lambda$ is proved in [14]. It is easy to check that the proof from [14] works also under our assumptions. Nevertheless we include here a short proof of Lemma 5 for the reader's convenience.

Proof. If $\chi_{b_{r}}$ is a characteristic function of Euclidean ball of radius $r$ centred at 0 in $\mathbf{R}^{Q}$, then function $\chi_{b_{r}} K_{F\left(\sqrt{\Delta_{Q}}\right)}$ and $\left(1-\chi_{b_{r}}\right) K_{F\left(\sqrt{\Delta_{Q}}\right)}$ are radial, so there exist functions $F_{r}: \mathbf{R}_{+} \rightarrow \mathbf{C}$ and $F^{r}: \mathbf{R}_{+} \rightarrow \mathbf{C}$ such that

$$
K_{F_{r}\left(\sqrt{\Delta_{Q}}\right)}=\chi_{b_{r}} K_{F\left(\sqrt{\Delta_{Q}}\right)}
$$

and

$$
K_{F^{r}\left(\sqrt{\Delta_{Q}}\right)}=\left(1-\chi_{b_{r}}\right) K_{F\left(\sqrt{\Delta_{Q}}\right)} .
$$

If $H$ is an even function which coincides with $F_{r}$ on $\mathbf{R}_{+}$, and $\hat{H}$ its Fourier transform, then

$$
\text { supp } \hat{H} \subset[-r, r],
$$

but by the property of the final speed of propagation of the wave equation, e.g. see [15] or [11] for proof, we have

$$
\operatorname{supp}\left(K_{C_{t}(\sqrt{L})}\right) \subset\{g:|g| \leq t\},
$$

where $C_{t}(\lambda)=\cos (t \lambda)$. Hence, using the functional calculus formula

$$
F_{r}(\sqrt{L})=\frac{1}{2 \pi} \int \hat{H}(t) C_{t}(\sqrt{L}) d t
$$

we get

$$
\operatorname{supp}\left(F_{r}(\sqrt{L})\right) \subset\{g \in G:|g| \leq r\} .
$$

Obviously $F=F_{r}+F^{r}$, so by $(26)$

$$
K_{F^{r}(\sqrt{L})}(g)=K_{F(\sqrt{L})}(g) \text { for }|g|>r .
$$

On the other hand, by the Plancherel Theorem (or by (9)), for any function $F$ : $\mathbf{R}_{+} \rightarrow \mathbf{C}$ we have

$$
\left\|K_{F\left(\sqrt{\Delta_{Q}}\right)}\right\|_{L^{2}}^{2}=C_{Q} \int_{0}^{\infty}|F(\lambda)|^{2} \lambda^{Q-1} d \lambda
$$


and by (23) we get

$$
\int_{G}\left|K_{F(\sqrt{L})}(g)\right|^{2} d g \leq C \int_{\mathbf{R}^{Q}}\left|K_{F\left(\sqrt{\Delta_{Q}}\right)}(x)\right|^{2} d x .
$$

Hence by virtue of $(27)$

$$
\begin{gathered}
\int_{|g|>r}\left|K_{F(\sqrt{L})}(g)\right|^{2} d g=\int_{|g|>r}\left|K_{F^{r}(\sqrt{L})}(g)\right|^{2} d g \leq \int_{G}\left|K_{F^{r}(\sqrt{L})}(g)\right|^{2} d g \\
\leq C \int_{\mathbf{R}^{Q}}\left|K_{F^{r}\left(\sqrt{\Delta_{Q}}\right)}(x)\right|^{2} d x=C \int_{|x|>r}\left|K_{F\left(\sqrt{\Delta_{Q}}\right)}(x)\right|^{2} d x .
\end{gathered}
$$

Finally, by (28), for any $a>0$

$$
\begin{aligned}
\int_{G}|g|^{a}\left|K_{F(\sqrt{L})}(g)\right|^{2} d g=\int_{G} \int_{0}^{|g|} a r^{a-1}\left|K_{F(\sqrt{L})}(g)\right|^{2} d r d g \\
\quad=\int_{0}^{\infty} a r^{a-1} \int_{|g|>r}\left|K_{F(\sqrt{L})}(g)\right|^{2} d r d g \leq C \int_{0}^{\infty} a r^{a-1} \int_{|x|>r}\left|K_{F\left(\sqrt{\Delta_{Q}}\right)}(x)\right|^{2} d x \\
=C \int_{\mathbf{R}^{Q}}|x|^{a}\left|K_{F\left(\sqrt{\Delta_{Q}}\right)}(x)\right|^{2} d x .
\end{aligned}
$$

This proves (24). To show (25) we note that

$$
\begin{aligned}
\int_{G}\left|X_{i} K_{F(\sqrt{L})}(g)\right|^{2} d g & \leq \int_{G}\left|\sqrt{L} K_{F(\sqrt{L})}(g)\right|^{2} d g \\
& \leq C \int_{\mathbf{R}^{Q+2}}\left|K_{F\left(\sqrt{\Delta_{Q+2}}\right)}(x)\right|^{2} d x
\end{aligned}
$$

and we prove (25) in a way similar to (24).

Using Theorem 1 and Lemma 5, we will prove the following version of Alexopoulos' multiplier theorem for quasi-homogeneous operators.

Theorem 2. Let $L$ be a quasi-homogeneous operator on a Lie group $G$ and let $d \leq D$ be the dimensions of $L$ at 0 and at infinity respectively. Let $\psi \in C_{c}^{\infty}\left(\mathbf{R}_{+}\right)$ be any fixed auxiliary function, not identically zero. If $F$ satisfies the condition

$$
\sup _{t>0}\left(\|\psi(\cdot) F(t \cdot)\|_{H_{d / 2+\varepsilon}}^{2}+(1+t)^{\frac{d-D}{2}}\|\psi(\cdot) F(t \cdot)\|_{H_{D / 2+\varepsilon}}^{2}\right)<\infty
$$

for some $\varepsilon>0$, then the operator $F(L)$ is bounded on $L^{p}$ for $1<p<\infty$ and of weak type $1 \rightarrow 1$.

Proof. (29) is actually independent of the choice of $\psi$, so we can assume that $\psi(\lambda) \in C_{c}^{\infty}([1,4])$ and

$$
\sum_{n=-\infty}^{\infty} \psi\left(2^{n} \lambda\right)=1 \text { for all } \lambda>0 .
$$

We put

$$
F_{n}(\lambda)=\psi\left(2^{-n} \lambda\right) F(\lambda)
$$


and

$$
\Psi_{n}(\lambda)=F_{n}\left(2^{n} \lambda\right)
$$

Note that

$$
F(L)=\sum_{n=-\infty}^{\infty} F_{n}(L)
$$

To prove Theorem 2 it is enough to show that

$$
\int_{|g|>r}\left|K_{F_{n}(L)}(g)\right| d g \leq C\left(r 2^{n / 2}\right)^{-\varepsilon}
$$

and

$$
\int_{G}\left|X_{i} K_{F_{n}(L)}(g)\right| d g \leq C 2^{n / 2} .
$$

Indeed, Theorem 2 follows by (30), (31) and (32) by standard Calderón-Zygmund analysis (see the proof of Theorem 7.9.5 in [8]).

Proof of (31) and (32). By (29),

$$
\left\|\Psi_{n}\right\|_{H_{d / 2+\varepsilon}}^{2} \leq C
$$

and

$$
\left\|\Psi_{n}\right\|_{H_{D / 2+\varepsilon}}^{2} \leq C\left(1+2^{n}\right)^{\frac{D-d}{2}} .
$$

By virtue of Theorem 1 and Lemma 5 ,

$$
\begin{gathered}
\int_{G}|g|^{s}\left|K_{F_{n}(L)}(g)\right|^{2} d g \leq C\left(\int_{\mathbf{R}^{D}}|x|^{s}\left|K_{F_{n}\left(\Delta_{D}\right)}(x)\right|^{2} d x \wedge \int_{\mathbf{R}^{d}}|x|^{s}\left|K_{F_{n}\left(\Delta_{d}\right)}(x)\right|^{2} d x\right) \\
=C\left(2^{n \frac{(D-s)}{2}} \int_{\mathbf{R}^{D}}|x|^{s}\left|K_{\Psi_{n}\left(\Delta_{D}\right)}(x)\right|^{2} d x \wedge 2^{n \frac{(d-s)}{2}} \int_{\mathbf{R}^{d}}|x|^{s}\left|K_{\Psi_{n}\left(\Delta_{d}\right)}(x)\right|^{2} d x\right) \\
\leq C^{\prime}\left(2^{\frac{n(D-s)}{2}} \wedge 2^{n \frac{(d-s)}{2}}\right)\left\|\Psi_{n}\right\|_{H_{s / 2}}^{2} .
\end{gathered}
$$

On the other hand, by the Schwarz inequality

$$
\begin{aligned}
\left(\int_{|g|>r}\left|K_{F_{n}(L)}(g)\right| d g\right)^{2} \leq & \int_{G}\left(|g|^{D+2 \varepsilon}+|g|^{d+2 \varepsilon}\right)\left|K_{F_{n}(L)}(g)\right|^{2} d g \\
& \times \int_{|g|>r}\left(|g|^{D+2 \varepsilon}+|g|^{d+2 \varepsilon}\right)^{-1} d g .
\end{aligned}
$$

By (36), (33) and (34)

$$
\begin{aligned}
& \int_{G}\left(|g|^{D+2 \varepsilon}+|g|^{d+2 \varepsilon}\right)\left|K_{F_{n}(L)}(g)\right|^{2} d g \\
& \leq C(\left(2^{-n \varepsilon} \wedge 2^{-n\left(\varepsilon+\frac{D-d}{2}\right)}\right)\left\|\Psi_{n}\right\|_{H_{D / 2+\varepsilon}}^{2} \\
&\left.\quad+\left(2^{-n \varepsilon} \wedge 2^{-n\left(\varepsilon+\frac{d-D}{2}\right)}\right)\left\|\Psi_{n}\right\|_{H_{d / 2+\varepsilon}}^{2}\right) \leq C^{\prime} 2^{-n \varepsilon} .
\end{aligned}
$$


Finally, if $B_{t}=\{g \in G:|g| \leq t\}$ and $V\left(B_{t}\right)$ is the volume of the ball $B_{t}$, then by Proposition IV.5.7 of [20] $V\left(B_{t}\right) \sim t^{d} \wedge t^{D}$, so

$$
\begin{array}{rl}
\int_{|g|>r}\left(|g|^{D+2 \varepsilon}+|g|^{d+2 \varepsilon}\right)^{-1} & d g \\
=\int_{r}^{\infty}\left[\frac{d}{d t}\left(\left(t^{D+2 \varepsilon}+t^{d+2 \varepsilon}\right)^{-1}\right)\right] V\left(B_{t}\right) d t \\
\leq C r^{-2 \varepsilon}
\end{array}
$$

and (31) follows by (37) and (38).

Next by virtue of $(25)$ for $n<0$

$$
\begin{aligned}
\int_{G} \mid X_{i} & K_{F_{n}(L)}(g)\left|d g=\int_{|g|<2^{-n / 2}}\right| X_{i} K_{F_{n}(L)}(g)\left|d g+\int_{|g| \geq 2^{-n / 2}}\right| X_{i} K_{F_{n}(L)}(g) \mid d g \\
\leq & V\left(B_{2^{-n / 2}}\right)^{1 / 2}\left(\int_{G}\left|X_{i} K_{F_{n}(L)}(g)\right|^{2} d g\right)^{1 / 2} \\
& +\left(\int_{G}\left(|g|^{D+2 \varepsilon}\right)\left|X_{i} K_{F_{n}(L)}(g)\right|^{2} d g\right)^{1 / 2}\left(\int_{|g| \geq 2^{-n / 2}}\left(|g|^{D+2 \varepsilon}\right)^{-1} d g\right)^{1 / 2} \\
\leq & C\left(2^{-n D / 4+n((D+2) / 4)}+2^{(-\varepsilon+1) n / 2+\varepsilon n / 2}\right)\left\|\Psi_{n}\right\|_{H_{D / 2+\varepsilon}} \leq C^{\prime} 2^{n / 2} .
\end{aligned}
$$

Finally, for $n>0$

$$
\begin{gathered}
\int_{G}\left|X_{i} K_{F_{n}(L)}(g)\right| d g=\int_{|g|<2^{-n / 2}}\left|X_{i} K_{F_{n}(L)}(g)\right| d g+\int_{1>|g| \geq 2^{-n / 2}}\left|X_{i} K_{F_{n}(L)}(g)\right| d g \\
+\int_{|g| \geq 1}\left|X_{i} K_{F_{n}(L)}(g)\right| d g \leq V\left(B\left(2^{-n / 2}\right)\right)^{1 / 2}\left(\int_{|g|<2^{-n / 2}}\left|X_{i} K_{F_{n}(L)}(g)\right|^{2} d g\right)^{1 / 2} \\
+\left(\int_{G}\left(|g|^{d+2 \varepsilon}\right)\left|X_{i} K_{F_{n}(L)}(g)\right|^{2} d g\right)^{1 / 2} \times\left(\int_{1>|g| \geq 2^{-n / 2}}\left(|g|^{d+2 \varepsilon}\right)^{-1} d g\right)^{1 / 2} \\
+\left(\int_{G}\left(|g|^{D+2 \varepsilon}\right)\left|X_{i} K_{F_{n}(L)}(g)\right|^{2} d g\right)^{1 / 2} \times\left(\int_{|g| \geq 1}\left(|g|^{D+2 \varepsilon}\right)^{-1} d g\right)^{1 / 2} \\
\leq\left(2^{n(d+2) / 4-n d / 4}+2^{(-\varepsilon+1) n / 2+\varepsilon n / 2}\left\|\Psi_{n}\right\|_{H_{d / 2+\varepsilon}}\right. \\
+2^{-n \frac{-2 \varepsilon+D-D+2}{4}}\left\|\Psi_{n}\right\|_{H_{D / 2+\varepsilon}} \leq C^{\prime} 2^{n / 2} .
\end{gathered}
$$

This proves (32) and finishes the proof of Theorem 2.

Remark 4. Although Theorem 2 is the strongest result of this type known to the author, the papers of Müller and Stein [12] and Hebisch [7] suggest that Theorem 2 is not optimal. 


\section{REFERENCES}

1. G. Alexopoulos, Spectral multipliers on Lie groups of polynomial growth, Proc. Amer. Math. Soc. (3) 120 (1994), 973-979 . MR 95j:22016

2. M. Christ, $L^{p}$ bounds for spectral multipliers on nilpotent groups, Trans. Amer. Math. Soc. 328 (1991), 73-81. MR 92k:42017

3. M. Christ, C.D. Sogge, The weak type $L^{1}$ convergence of eigenfunction expansions for pseudodifferential operators, Invent. Math. 94 (1988), 421-453. MR 89j:35096

4. E.B. Davies, Heat Kernels and Spectral Theory, Cambridge University Press, 1989. MR 90e: 35123

5. A.F.M. ter Elst, D.W. Robinson, Subcoercivity and subelliptic operators on Lie groups II: The general case, Potential Analysis 4 (1995), 205-243. MR 98c:22007

6. G.B. Folland, E.M. Stein, Hardy Spaces on Homogeneous Groups, Princeton University Press, 1982. MR 84h:43027

7. W. Hebisch, Multiplier theorem on generalized Heisenberg groups, Colloq. Math. 65 (1993), 231-239. MR 93m:43013

8. L. Hörmander, The Analysis of Linear Partial Differential Operators I, Springer-Verlag, 1983. MR 85g:35002

9. L. Hörmander, On the Riesz means of spectral functions and eigenfunction expansions for elliptic differential operators, in: Some recent advances in the basic sciences, Yeshiva Univ., New York (1966), 155-202. MR 41:2239

10. L. Hörmander, The spectral function of an elliptic operator, Acta Math. 88 (1968), 193-218. MR 58:29418

11. R. Melrose, Propagation for the wave group of a positive subelliptic second order differential operator, in: Hyperbolic Equations and Related Topics, Academic Press, Boston, Mass. (1986), 181-192. MR 89h:35177

12. D. Müller, E.M. Stein, On spectral multipliers for Heisenberg and related groups, J. Math. Pures Appl. 73 (1994), 413-440. MR 96m:43004

13. A. Pazy Semigroups of Linear Operators and Applications to Partial Differential Equations, Springer-Verlag, 1983. MR 85g:47061

14. A. Sikora Multiplicateurs associés aux souslaplaciens sur les groupes homogènes, C. R. Acad. Sci. Paris Série I 315 (1992), 417-419. MR 93e:43010

15. A. Sikora, Sharp pointwise estimates on heat kernels, Quart. J. Math. Oxford (2) 47 (1996), 371-382. MR 97m:58189

16. C.D. Sogge, On the convergence of Riesz means on compact manifolds, Ann. of Math. 126 (1987), 439-447. MR 89b:35126

17. C.D. Sogge, Oscillatory integrals and spherical harmonics, Duke Math. J. 53 (1986), 43-65. MR 87g: 42026

18. C.D. Sogge, Concerning the $L^{p}$ norm of spectral clusters for second order elliptic operators on compact manifolds, J. Funct. Anal. 77 (1988), 123-138. MR 89d:35131

19. E.M. Stein, Harmonic Analysis: Real-Variable Methods, Orthogonality, and Oscillatory Integrals, Princeton University Press, 1993. MR 95c:42002

20. N.T. Varopoulos, L. Saloff-Coste, Th. Coulhon, Analysis and Geometry on Groups, Cambridge University Press, 1992. MR 95f:43008

Centre for Mathematics and Its Applications, School of Mathematical Sciences, Australian National University, Canberra ACT 0200, Australia

E-mail address: sikora@maths.anu.edu.au, sikora@math.uni.wroc.pl 